\title{
GANHOS DE EFICIÊNCIA FERTILIZANTE EM BANANEIRA SOB IRRIGAÇÃO E FERTIRRIGAÇÃO ${ }^{1}$
}

\author{
LUIZ ANTONIO JUNQUEIRA TEIXEIRA², JOSÉ ANTONIO QUAGGIO², \\ ESTÊVÃO VICARI MELLIS ${ }^{2}$
}

RESUMO - O aumento da eficiência fertilizante tem sido cada vez mais almejado nas boas práticas de manejo da adubação de culturas, não somente buscando reduzir custos de produção, mas também reduzir possíveis impactos ambientais. Com o objetivo de avaliar a eficiência dos fertilizantes nitrogenados e potássicos no cultivo da bananeira, foram realizados dois experimentos de campo com a cultivar 'Nanicão' (Musa AAA, subgrupo Cavendish), no Planalto do Estado de São Paulo, durante quatro ciclos de produção. Esta região caracteriza-se por estação chuvosa no verão e seca no inverno. No primeiro experimento, avaliou-se a eficiência da aplicação das doses de N: 0; 200; 400 e $800 \mathrm{~kg} \mathrm{ha}^{-1}$ e de K: 0; 300; 600 e $900 \mathrm{~kg} \mathrm{ha}^{-1}$ de $\mathrm{K}_{2} \mathrm{O}$, em sistema de cultivo irrigado, comparado ao de sequeiro. No segundo experimento, estudou-se a eficiência da adubação com N e K aplicada por fertirrigação e de modo convencional via solo. Nos dois experimentos, a irrigação e a fertirrigação foram feitas por microaspersão, empregando-se como fontes de $\mathrm{N}$ e de $\mathrm{K}$ nitrato de amônio e cloreto de potássio, respectivamente. A eficiência dos fertilizantes foi estimada pela relação entre a massa dos frutos produzidos por unidade de nutriente aplicado. Para a média das doses empregadas e dos ciclos de produção, observou-se que a irrigação incrementou a eficiência da adubação em relação ao sequeiro, em aproximadamente $36 \%$ e $32 \%$, respectivamente, para nitrogênio e potássio. A aplicação de nitrogênio e potássio via fertirrigação promoveu aumento de $36 \%$ na eficiência fertilizante em relação à adubação sólida convencional, via solo. Incrementos na eficiência dos fertilizantes decorrentes da irrigação e da fertirrigação possibilitaram diminuir os gastos com o uso destes insumos.

Termos para indexação: Eficiência de adubação, nitrogênio, potássio, banana.

\section{ENHANCING NUTRIENT USE EFFICIENCY IN BANANA TREE UNDER IRRIGATION AND FERTIGATION}

\begin{abstract}
The best nutritional practices aim to increase nutrient use efficiency in order to reduce production costs and environmental impacts. In order to evaluate nitrogen and potassium use efficiency in banana crops, two field experiments were carried out during four cycles of production. The experiments were cropped with Giant Cavendish banana (Musa AAA, Cavendish subgroup) in Planalto Region of State of Sao Paulo, Brazil. This region is characterized by a summer rainy season and dry winter. In the first experiment, the efficiency of the rates of $\mathrm{N}\left(0,200,400\right.$ and $\left.800 \mathrm{~kg} \mathrm{ha}^{-1}\right)$ and $\mathrm{K}\left(0,300,600\right.$ and $900 \mathrm{~kg} \mathrm{ha}^{-1}$ of $\mathrm{K}_{2} \mathrm{O}$ ) were compared in banana grown in irrigated and non-irrigated plots. In the second experiment, was compared $\mathrm{N}$ and $\mathrm{K}$ fertilizer efficiency applied through fertigation and conventional solid fertilization. In both experiments, irrigation and fertigation were made by micro-sprinkler and employed ammonium nitrate and potassium chloride as a source of $\mathrm{N}$ and $\mathrm{K}$, respectively. The fertilizer efficiency was estimated by the ratio of the mass of fruit produced per unit of applied nutrient. The results of polled harvest have shown that irrigation increased the nutrient use efficiency compared to non-irrigated, for the averaged nutrient rates, close to $36 \%$ and $32 \%$ for $\mathrm{N}$ and $\mathrm{K}$, respectively. Fertigation promoted an increase of $36 \%$ in nutrient use efficiency compared to conventional fertilization, for either nitrogen or potassium. Increases in nutrient use efficiency contributed to reduce fertilization costs.
\end{abstract}

Index terms: nutrient use efficiency; nitrogen; potassium; banana.

(Trabalho 030-10). Recebido em: 14-01-2010. Aceito para publicação em: 28-09-2010. Trabalho realizado com o apoio da FAPESP (Projetos 96/4209-4 e 01/09976-3).

${ }^{2}$ Pesquisadores do Centro de Solos e Recursos Ambientais do Instituto Agronômico (IAC). Caixa Postal 28. 13001-970, Campinas-SP, Brasil. teixeira@iac.sp.gov.br, quaggio@iac.sp.gov.br, evmellis@iac.sp.gov.br 


\section{INTRODUÇÃO}

O Estado de São Paulo é um dos maiores produtores nacionais de bananas, colhendo, em 2008, cerca de 1,2 milhão de toneladas de frutos/ano em 60 mil hectares, dos quais aproximadamente $70 \%$ encontram-se no Vale do Ribeira (IEA, 2010). Nos últimos anos, houve forte expansão da bananicultura para o Planalto Paulista em áreas cultivadas anteriormente com café e pastagem em busca de maior rentabilidade com a fruticultura. Entretanto, a expansão da bananicultura para o Planalto implica necessidade de irrigação, pois esta região caracterizase por apresentar acentuado déficit hídrico durante o inverno (TEIXEIRA, 2008). A inadequação do pacote tecnológico para o cultivo no Planalto Paulista começa pelas recomendações de adubação para a bananeira no Estado de São Paulo, as quais foram definidas com base em resultados de pesquisa obtidos principalmente na região do Vale do Rio Ribeira, onde a irrigação não é prática usual. Além disso, a informação disponível sobre resposta da bananeira à irrigação e à fertirrigação no Planalto Paulista é incipiente na literatura brasileira.

Quando se discute agricultura intensiva, a irrigação e a adubação são condições essenciais para sua implementação. A interação existente entre esses fatores faz a resposta das culturas à aplicação de fertilizantes ser fortemente alterada pelo regime hídrico do solo. Bar-Yosef (1999) acrescenta que a irrigação e a fertilização seriam os fatores passíveis de manejo mais importantes para o controle do desenvolvimento das plantas, do rendimento e da qualidade de frutos.

A dinâmica do nitrogênio e do potássio no sistema solo-planta é fortemente condicionada pela água disponível (HAVLIN et al., 2005). Segundo Olson (1984), a água disponível no solo é essencial para que as plantas respondam à aplicação de $\mathrm{N}$; ao mesmo tempo, elas devem estar adequadamente nutridas com $\mathrm{N}$ para utilizarem eficientemente a água do solo. Além disso, a água afeta desde os mecanismos de transporte do $\mathrm{N}$ no sistema solo-planta, até o desenvolvimento vegetativo, o que determina as respostas à adubação nitrogenada.

No caso do potássio, sua disponibilidade é largamente determinada pelas condições de difusão e de fluxo de massa no solo, que, por sua vez, são funções do seu conteúdo de água. Thomas e Bertsch (1985) afirmaram que a umidade é um fator crítico para o movimento do K no solo e sua absorção pelas plantas. Barber (1995) apresentou o efeito da umidade na difusão e disponibilidade de íons no solo. Vários autores, como Hylander et al. (1999), trabalhando com soja, algodão, milho e arroz, e Oliveira et al. (2004), com algodão, demonstraram este efeito experimentalmente.

Revisando resultados de pesquisa com fertirrigação em bananeira, Natale e Rodrigues (2006) mencionaram que esta forma de aplicação dos nutrientes possibilita aumentar tanto a produtividade como a qualidade dos frutos, com redução do custo operacional e melhora na eficiência dos fertilizantes.

O objetivo deste trabalho foi estudar a eficiência da adubação nitrogenada e potássica em bananeira 'Nanicão' em função do regime hídrico (sequeiro e sob irrigação), bem como comparar a eficiência desses fertilizantes aplicados por meio da adubação convencional, via solo e via fertirrigação.

\section{MATERIAL E MÉTODOS}

Foram executados durante quatro ciclos de produção dois experimentos de campo com bananeira 'Nanicão' (Musa AAA subgrupo Cavendish), no Planalto do Estado de São Paulo. O primeiro experimento localizou-se em Jaboticabal (Altitude: $575 \mathrm{~m} ; 21^{\circ} 15^{\prime} \mathrm{S} ; 48^{\circ} 18^{\prime} \mathrm{W}$ ), num solo classificado como Latossolo Vermelho eutroférrico típico ( $E u$ trustox). O segundo foi realizado em Pindorama (Altitude: $530 \mathrm{~m} ; 21^{\circ} 13^{\prime} \mathrm{S} ; 48^{\circ} 55^{\prime} \mathrm{W}$ ), num Argissolo Vermelho-Amarelo eutrófico (Ultisol). O clima da região, conforme a classificação de Köeppen, é do tipo Aw, definido como tropical úmido, com estação chuvosa no verão e seca no inverno. $\mathrm{O}$ balanço hídrico indica que o período mais seco está entre os meses de julho e setembro, enquanto o período de maior excedente hídrico compreende os meses de janeiro a março. Detalhes das áreas experimentais, bem como do manejo das plantas, estão descritos em Teixeira et al. (2002) e Teixeira et al. (2007).

No primeiro experimento, foram avaliados os efeitos da aplicação de $\mathrm{N}\left(0 ; 200 ; 400\right.$ e $\left.800 \mathrm{~kg} \mathrm{ha}^{-1}\right)$, de potássio $\left(0 ; 300 ; 600\right.$ e $900 \mathrm{~kg} \mathrm{ha}^{-1}$ de $\left._{2} \mathrm{O}\right)$ e do regime hídrico (sequeiro e irrigado), utilizando-se de um arranjo fatorial $2 \times 4 \times 4$. No segundo ensaio, foram empregadas quatro doses de N $(0 ; 140 ; 280$ e 420 $\left.\mathrm{kg} \mathrm{ha}^{-1}\right)$ e de $\mathrm{K}\left(0 ; 160 ; 320\right.$ e $480 \mathrm{~kg} \mathrm{ha}^{-1}$ de $\left.\mathrm{K}_{2} \mathrm{O}\right)$, com os fertilizantes sendo aplicados via fertirrigação ou adubação sólida convencional. Nesse experimento, os tratamentos foram estabelecidos a partir da recomendação de adubação para a bananeira no Estado de São Paulo (TEIXEIRA et al., 1997), sendo estudadas cinco frações desta recomendação $(0 ; 40$ $\%, 80 \%$ e $120 \%$ da recomendação). Nos dois experimentos, utilizou-se, como fonte de $\mathrm{N}$ e de $\mathrm{K}$, nitrato de amônio e cloreto de potássio, respectivamente.

A irrigação e a fertirrigação foram feitas por microaspersão. No primeiro experimento (regime 
hídrico), foram empregados emissores com vazão de 50 L/hora; no segundo (fertirrigação), os emissores tinham vazão de $60 \mathrm{~L} /$ hora. A irrigação foi manejada a partir de dados meteorológicos (evaporação medida no tanque classe A e precipitação), coletados próximos às áreas experimentais, visando a atender às exigências hídricas da cultura, estimadas de acordo com Doorenbos e Kassam (1979). Em ambos os experimentos, adotou-se intervalo entre regas de dois ou três dias. No experimento um, no qual foram comparados regimes hídricos, as doses de $\mathrm{N}$ e $\mathrm{K}$ foram fracionadas em quatro aplicações durante a época das chuvas. No experimento dois, a fertirrigação foi realizada quinzenalmente. Em ambos os experimentos, utilizaram-se mudas micropropagadas estabelecidas no espaçamento de 2 X 2,5 m (2.000 plantas ha-1). Para o plantio e práticas de manejo do bananal, foram seguidas as recomendações descritas por Moreira (1999).

A eficiência de aplicação dos fertilizantes foi avaliada por meio da relação entre a massa dos frutos produzidos por unidade de nutriente aplicada, ou seja, $\mathrm{kg}$ de frutos por $\mathrm{kg}$ do nutriente aplicado. Segundo Dobermann (2007), este índice é importante sob o ponto de vista dos produtores, pois integra, simultaneamente, os conceitos de eficiência de uso dos nutrientes provenientes dos fertilizantes e o da mobilização de reservas do solo. E sob o aspecto científico, esta forma de estimar a eficiência dos fertilizantes é a mais adequada quando os tratamentos-controle (sem N) apresentam produtividades muito diferentes em função de regime hídrico ou de manejo.

A partir do cálculo da eficiência dos fertilizantes, estimou-se o custo de aquisição dos fertilizantes para produzir $40 \mathrm{t}$ de frutos por hectare nas diversas condições de manejo, sendo esta despesa expressa em $\mathrm{kg}$ de frutos. Nesta estimativa de custo, foram computadas as eficiências dos fertilizantes para cada forma de aplicação e regime hídrico e os preços médios de $\mathrm{N}, \mathrm{K}_{2} \mathrm{O}$ (preços pagos pelos agricultores, sem custo de aplicação) e dos frutos praticados no Estado de São Paulo nos últimos cinco anos (IEA, 2010). Nos dois experimentos, foram consideradas as médias de produção dos ciclos e as quantidades de fertilizantes aplicadas por ciclo da cultura.

As médias de eficiência dos fertilizantes em função do regime hídrico (irrigado e sequeiro) e da forma de aplicação dos fertilizantes (convencional e fertirrigação) foram comparadas por meio do teste-t de Student, sendo considerados significantemente diferentes valores com $p<0,05$.

\section{RESULTADOS E DISCUSSÃO}

As médias, amplitudes e desvios-padrão das doses de fertilizantes, da produção das bananeiras e dos índices de eficiência de aplicação dos fertilizantes obtidos nas duas áreas experimentais são apresentados na Tabela 1. Tanto a produção como a eficiência dos adubos aplicados apresentaram ampla variação, o que torna o banco de dados interessante para a avaliação da eficiência dos fertilizantes sob diversas condições de manejo dos bananais.

A eficiência dos fertilizantes nitrogenados e potássicos variou em função do regime hídrico (Tabela 2). A irrigação proporcionou eficiência significativamente maior do que em condições de sequeiro, tanto para a adubação nitrogenada, como para a potássica. $\mathrm{O}$ incremento médio na eficiência da adubação nitrogenada decorrente da irrigação foi de aproximadamente $36 \%$, enquanto para a potássica a eficiência aumentou em $32 \%$. Os mecanismos de movimentação dos nutrientes no solo, sua absorção pelas plantas, bem como o desenvolvimento vegetativo, provavelmente, foram positivamente influenciados pela disponibilidade hídrica. Com o aumento das doses de $\mathrm{N}$ e $\mathrm{K}$, a eficiência dos fertilizantes diminuiu, refletindo a "lei dos retornos decrescentes" como apontado por Havlin et al. (2005). Estes resultados são coerentes com aqueles obtidos por Quaggio et al. (2006), indicando que a irrigação e a fertirrigação aumentam a eficiência de uso de $\mathrm{N}$ em laranjeiras, em 12 e $25 \%$, respectivamente, em relação à adubação sólida convencional.

A adubação via fertirrigação também favoreceu aumentos de eficiência em relação à adubação sólida convencional (Tabela 3). Os incrementos na eficiência da adubação com $\mathrm{N}$ e $\mathrm{K}$ foram de aproximadamente $36 \%$ quando os fertilizantes foram aplicados via fertirrigação. Stewart et al. (1998) afirmaram que a fertirrigação em bananeira aumenta a eficiência do uso de fertilizantes, possibilitando reduzir as doses recomendadas para aplicação convencional, entre 20 e $30 \%$. As principais vantagens da fertirrigação decorrem de que a aplicação de fertilizantes de forma mais fracionada implica que a disponibilidade dos nutrientes se mantenha menos variável ao longo do ciclo e que eventuais precipitações de alta intensidade, causadoras de erosão ou lixiviação, tenham menor impacto sobre o crescimento das plantas. Os fertilizantes na água de irrigação são aplicados nos locais de maior absorção e prontamente acessíveis às plantas. Perdas gasosas ou por escorrimento superficial também são minimizadas com a fertirrigação. Na Índia, Srinivas (1997) obteve rendimento de frutos semelhante (em torno 
de 30 t ha $^{-1}$ ) com aplicação de $100 \mathrm{~g} /$ planta de $\mathrm{N}$ via fertirrigação e com $200 \mathrm{~g} /$ planta de $\mathrm{N}$ aplicados ao solo. Para Hagin e Tucker (1982), maior aproveitamento de $\mathrm{N}$ por bananeiras foi obtido com ureia aplicada por fertirrigação em relação à aplicação convencional na superfície do solo.

Relatos de maior eficiência dos fertilizantes aplicados via fertirrigação também são comuns para outras frutíferas, como laranja em Israel (DASBERG et al., 1988) e pomelo na Flórida (BOMAN, 1996). Quaggio et al. (2007), em trabalho semelhante com laranjeiras, observaram, através de estudos de equilíbrio químico da solução do solo, que o ganho de eficiência de uso de $\mathrm{N}$ da fertirrigação foi relacionado às maiores concentrações do nutriente na solução do solo, proporcionadas pela fertirrigação em relação à adubação sólida, o que favoreceu o processo de absorção do nutriente pela planta.

Ainda que o aumento da eficiência dos fertilizantes seja importante para minimizar o impacto ambiental da atividade agrícola, do ponto de vista dos produtores, este incremento torna-se mais atraente se apresentar algum retorno econômico. Nas Tabelas $4 \mathrm{e}$ 5 , são apresentadas estimativas de redução de gastos com fertilizantes em função da irrigação e da fertirrigação, respectivamente. Estas estimativas referem-se a todo o conjunto de doses estudadas em cada um dos experimentos e serve para ilustrar o potencial de economia decorrente do aumento da eficiência dos fertilizantes empregados em bananeira quando são adotadas práticas agronômicas adequadas. Entre essas práticas, destacam-se a irrigação e a fertirrigação devido às altas exigências nutricionais da cultura e às condições climáticas do Planalto Paulista.

TABELA 1- Dados coletados nas áreas experimentais com bananeira 'Nanicão' no Planalto Paulista, Brasil.

\begin{tabular}{|c|c|c|c|c|}
\hline Variável & Mínimo & Média & Máximo & Desvio-padrão \\
\hline \multicolumn{5}{|c|}{----------------- Experimento 1 (Jaboticabal-SP) ---------------- } \\
\hline Dose de $\mathrm{N}\left(\mathrm{kg} \mathrm{ha}^{-1}\right)$ & 200 & 467 & 800 & -- \\
\hline Dose de $\mathrm{K}\left(\mathrm{kg} \mathrm{ha}^{-1}\right.$ de $\left.\mathrm{K}_{2} \mathrm{O}\right)$ & 300 & 600 & 900 & -- \\
\hline Produção por ciclo ( $\left.\mathrm{t} \mathrm{ha}^{-1}\right)$ & 17,7 & 34,2 & 66,1 & 11,8 \\
\hline Índice de $\mathrm{N}^{(1)}$ & 22,9 & 101,1 & 330,4 & 69,7 \\
\hline Índice de $\mathrm{K}^{(2)}$ & 22,5 & 69,2 & 200,1 & 40,9 \\
\hline \multicolumn{5}{|c|}{ 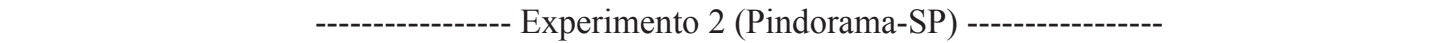 } \\
\hline Dose de $\mathrm{N}\left(\mathrm{kg} \mathrm{ha}^{-1}\right)$ & 140 & 308 & 420 & -- \\
\hline Dose de $\mathrm{K}\left(\mathrm{kg} \mathrm{ha}^{-1}\right.$ de $\mathrm{K}_{2} \mathrm{O}$ & 160 & 352 & 480 & -- \\
\hline Produção por ciclo $\left(\mathrm{t} \mathrm{ha}^{-1}\right)$ & 14,0 & 44,1 & 73,6 & 13,9 \\
\hline Índice de $\mathrm{N}^{(1)}$ & 40,0 & 167,6 & 442,9 & 96,8 \\
\hline Índice de $\mathrm{K}^{(2)}$ & 35,0 & 146,6 & 387,5 & 84,7 \\
\hline
\end{tabular}

(1) Índice de $\mathrm{N}=\mathrm{kg}$ de fruto/kg de $\mathrm{N}$ aplicado. ${ }^{(2)}$ Índice de $\mathrm{K}=\mathrm{kg}$ de fruto $/ \mathrm{kg}$ de $\mathrm{K}_{2} \mathrm{O}$ aplicado.

TABELA 2 - Índice de eficiência fertilizante de doses de N e de K aplicados em bananeira 'Nanicão' em função do regime hídrico (Experimento 1).

\begin{tabular}{|c|c|c|c|}
\hline Dose de N $\left(\mathrm{kg} \mathrm{ha}^{-1}\right)$ & Irrigado & Sequeiro & Teste-t para médias \\
\hline & \multicolumn{2}{|c|}{--------- Índice de $\mathrm{N}^{(1)}$--------- } & Valor $p^{(3)}$ \\
\hline 200 & 197,7 & 157,2 & 0,059 \\
\hline 400 & 96,3 & 72,3 & 0,015 \\
\hline 800 & 48,4 & 34,9 & 0,014 \\
\hline Média três doses & 113,4 & 83,5 & $\mathbf{0 , 0 1 6}$ \\
\hline \multicolumn{3}{|l|}{ Dose de K $\left(\mathrm{kg} \mathrm{ha}^{-1}\right.$ de $\left._{2} \mathrm{O}\right)$} & \\
\hline 300 & 126,9 & 97,2 & 0,031 \\
\hline 600 & 64,7 & 49,0 & $\mathbf{0 , 0 3 5}$ \\
\hline 900 & 44,3 & 33,0 & 0,013 \\
\hline Média três doses & 77,2 & 58,4 & 0,008 \\
\hline
\end{tabular}

(1) Índice de $\mathrm{N}=\mathrm{kg}$ de fruto/kg de $\mathrm{N}$ aplicado. ${ }^{(2)}$ Índice de $\mathrm{K}=\mathrm{kg}$ de fruto/ $\mathrm{kg}$ de $\mathrm{K}_{2} \mathrm{O}$ aplicado. ${ }^{(3)}$ Nível de significância do teste-t; valores em negrito são significativos a $5 \%$ de probabilidade. $(p<0,05)$. 
TABELA 3 - Índice de eficiência fertilizante para N e K aplicados em bananeira 'Nanicão' em função da forma de aplicação dos nutrientes (Experimento 2).

\begin{tabular}{cccc}
\hline Variável & Fertirrigação & Adubação via solo & Teste-t para médias \\
\hline & & & Valor $p^{(1)}$ \\
Índice de $\mathrm{N}^{(2)}$ & 205,6 & 150,9 & $\mathbf{0 , 0 0 3}$ \\
${\text { Índice de } \mathrm{K}^{(3)}}^{13}$ & 179,9 & 132,0 & $\mathbf{0 , 0 0 3}$ \\
\hline
\end{tabular}

(1) Nível de significância do teste-t; valores em negrito são significativos a $5 \%$ de probabilidade. $(p<0,05)$. ${ }^{(2)}$ Índice de $\mathrm{N}=\mathrm{kg}$ de fruto/kg de $\mathrm{N}$ aplicado. ${ }^{(3)}$ Índice de $\mathrm{K}=\mathrm{kg}$ de fruto/kg de $\mathrm{K}_{2} \mathrm{O}$ aplicado.

TABELA 4 - Custo (equivalente em $\mathrm{kg}$ de frutos) dos fertilizantes nitrogenados e potássicos aplicados em bananeira 'Nanicão', visando à produção de $40 \mathrm{t}$ de frutos/hectare, em função do regime hídrico no Planalto Paulista, Brasil.

\begin{tabular}{|c|c|c|c|c|c|}
\hline \multirow{2}{*}{ Nutriente } & \multirow{2}{*}{ Irrigado } & \multirow{2}{*}{ Sequeiro } & \multirow{2}{*}{$\begin{array}{c}\text { Economia devido } \\
\text { à irrigação }\end{array}$} & \multicolumn{2}{|c|}{ Doses para produzir $40 \mathrm{t} \mathrm{ha}^{-1}$} \\
\hline & & & & Irrigado & Sequeiro \\
\hline \multicolumn{6}{|c|}{ Custo do fertilizante por hectare em kg de frutos ${ }^{(1)}$} \\
\hline $\mathrm{N}$ & 2087 & 2835 & 748 & $353^{(2)}$ & $479^{(2)}$ \\
\hline $\mathrm{K}$ & 2435 & 3219 & 784 & $518^{(3)}$ & $685^{(3)}$ \\
\hline $\mathrm{N}+\mathrm{K}$ & 4522 & 6054 & 1532 & -- & -- \\
\hline
\end{tabular}

Custo (equivalente em $\mathrm{kg}$ de frutos) do fertilizante aplicado ( $\mathrm{N}$ ou $\mathrm{K}$ ) visando à produção de $40 \mathrm{t}$ de frutos/ha. Estımativa baseada $\left.\mathrm{K}_{2} \mathrm{O}\right)$ e frutos $\left(\mathrm{R} \$ 0,51 / \mathrm{kg}\right.$ de frutos) nos últimos cinco anos (IEA, 2010). ${ }^{(2)}$ Dose de $\mathrm{N}\left(\mathrm{kg} \mathrm{ha}^{-1}\right)$ estimada visando à produção de $40 \mathrm{t}$ de frutos/ha. ${ }^{(3)}$ Dose de $\mathrm{K}\left(\mathrm{kg} \mathrm{ha}^{-1} \mathrm{de}_{2} \mathrm{O}\right)$ estimada visando à produção de $40 \mathrm{t}$ de frutos/ha.

TABELA 5 - Custo (equivalente em kg de frutos) dos fertilizantes nitrogenados e potássicos aplicados em bananeira 'Nanicão', visando à produção de $40 \mathrm{t}$ de frutos/hectare, em função da forma de aplicação dos fertilizantes no Planalto Paulista, Brasil.

\begin{tabular}{|c|c|c|c|c|c|}
\hline \multirow{2}{*}{ Nutriente } & \multirow{2}{*}{ Fertirrigação } & \multirow{2}{*}{$\begin{array}{c}\text { Convencional } \\
\text { via solo }\end{array}$} & \multirow{2}{*}{$\begin{array}{c}\text { Economia devido à } \\
\text { fertirrigação }\end{array}$} & \multicolumn{2}{|c|}{ Doses para produzir $40 \mathrm{t} \mathrm{ha}^{-1}$} \\
\hline & & & & Fertirrigação & Conv. via solo \\
\hline & \multicolumn{5}{|c|}{ Custo do fertilizante por hectare em $\mathrm{kg}$ de frutos ${ }^{(1)}$} \\
\hline $\mathrm{N}$ & 1151 & 1569 & 418 & $195^{(2)}$ & $265^{(2)}$ \\
\hline $\mathrm{K}$ & 1045 & 1424 & 379 & $222^{(3)}$ & $303^{(3)}$ \\
\hline $\mathrm{N}+\mathrm{K}$ & 2196 & 2993 & 797 & -- & -- \\
\hline
\end{tabular}

${ }^{(1)}$ Custo (equivalente em $\mathrm{kg}$ de frutos) do fertilizante aplicado $(\mathrm{N}$ ou $\mathrm{K}$ ) visando à produção de $40 \mathrm{t}$ de frutos/ha. Estimativa baseada nos índices de eficiência dos fertilizantes nas duas formas de aplicação e nos preços médios de $\mathrm{N}$ ( $\mathrm{R} \$ 3,02 / \mathrm{kg}$ de $\mathrm{N}$ ), $\mathrm{K}_{2} \mathrm{O}$ ( $\mathrm{R} \$ 2,40 / \mathrm{kg}$ de $\mathrm{K}_{2} \mathrm{O}$ ) e frutos (R\$ 0,51/kg de frutos) nos últimos cinco anos (IEA, 2010). ${ }^{(2)}$ Dose de $\mathrm{N}\left(\mathrm{kg} \mathrm{ha}^{-1}\right)$ estimada visando à produção de 40 $\mathrm{t}$ de frutos/ha. ${ }^{(3)}$ Dose de $\mathrm{K}\left(\mathrm{kg} \mathrm{ha}^{-1}\right.$ de $\left.\mathrm{K}_{2} \mathrm{O}\right)$ estimada visando à produção de $40 \mathrm{t}$ de frutos/ha. 


\section{CONCLUSÕES}

1-A eficiência dos fertilizantes nitrogenados e potássicos aplicados em bananeira 'Nanicão' aumenta com a irrigação.

2-Em área irrigada, a aplicação de fertilizantes nitrogenados e potássicos é mais eficiente quando feita por meio da fertirrigação.

3-Incrementos na eficiência dos fertilizantes decorrentes da irrigação e da fertirrigação possibilitam minimizar os gastos com o uso destes insumos.

\section{REFERÊNCIAS}

BARBER, S.A. Soil nutrient biovailability: a mechanistic approach. $2^{\text {nd }}$ ed. New York: John \&Wiley, 1995. 414p.

BAR-YOSEF, B. Advances in fertigation. In: SPARKS, D.L. (Ed.). Advances in Agronomy. New York: Academic Press, 1999. p.1-77.

BOMAN, B.J. Fertigation versus conventional fertilization of flatwood grapefruit. Fertilizer Research, The Hague, v.44, p.123-128, 1996.

DASBERG, S.; BAR-AKIVA, A.; SPAZISKY, S.; COHEN, A. Fertigation versus broadcasting in an orange grove. Fertilizer Research, The Hague, v.15, p. 147-154, 1988.

DOBERMANN, A. Nutrient use efficiency: measurement and management. In. INTERNATIONAL FERTILIZER INDUSTRY ASSOCIATION. Fertilizer best management practices. Paris, 2007. p.1-28.

DOORENBOS, J.; KASSAN, A.H. Yield response to water. Roma: FAO, 1979. 193 p. (Irrigation and Drainage Paper, 33)

HAGIN, J.; TUCKER, B. Fertilization of dryland and irrigated soils. Berlin: Springer-Verlag, 1982. 190p.

HAVLIN, J.L.; BEATON, J.D.; TISDALE, S.L.; NELSON, W.L. Soil fertility and fertilizers. 7. ed. Upper Saddle River: Pearson Education, 2005. 515p.
HYLANDER, L.D.; AE, N.; HATTA, T.; SUGIYAMA, M. Exploitation of $\mathrm{K}$ near roots of cotton, maize, upland rice and soybean grown in an Ultisol. Plant Soil, Dordrecht, v.208, p.33-41, 1999.

IEA. Instituto de Economia Agrícola. Disponível em: $<$ http://www.iea.sp.gov.br>. Acesso em: 11 jan. 2010.

MOREIRA, R.S. Banana: teoria e prática de cultivo. 2.ed. São Paulo: Fundação Cargill, 1999. CD-ROM

NATALE, W.; RODRIGUES, M.G.V. Fertirrigação em Bananeira. In: BOARETTO, A.E.; VILLAS BOAS, R.L.; SOUZA, W.F.; PARRA,L.R.V. (Ed.). Fertirrigação: teoria e prática. Piracicaba, 2006. v.1, p.396-439. CD-ROM

OLIVEIRA, R. H.; ROSOLEM, C. A.; TRIGUEIRO, R. M. Importância do fluxo de massa e difusão no suprimento de potássio ao algodoeiro como variável de água e potássio no solo. Revista Brasileira de Ciência do Solo, Viçosa, MG, v.28, p.439-445, 2004.

OLSON, R.A. Nitrogen use in dryland farming under semiarid conditions. In: HAUCK, R.D. (Ed.). Nitrogen in crop production. Madison: ASA/CSSA/ SSSA, 1984. p.335-347.

QUAGGIO, J.A.; MATTOS JR., D. ; SOUZA, T.R.; BOARETTO, R.M. Equilíbrio químico na solução do solo em sistemas de adubação sólida e fertirrigação na citricultura. In: CONGRESSO BRASILEIRO DE CIÊNCIA DO SOLO, 32., 2007. Gramado. Anais... CD-ROM

QUAGGIO, J.A.; SOUZA, T.R. ; MATTOS JR., D.; MENEZES, G.; KRONTAL, Y. Dinâmica de íons no solo e ganhos de eficiência fertilizante devido à irrigação e fertirrigação na citricultura. In: REUNIÃO BRASILEIRA DE FERTILIDADE E MICROBIOLOGIA DO SOLO- FERTBIO, 2006, Bonito. Anais... CD-ROM.

SRINIVAS, K. Growth, yield, and quality of banana in relation to $\mathrm{N}$ fertigation. Tropical Agriculture, Trinidad, v.74, n.4, p.260-264, 1997.

STEWART, L. et al. Tropical banana information kit. Nambour: Queensland Department of Primary Industries, 1998. (Serie Agrilink) 
TEIXEIRA, L.A.J. Bananicultura no Planalto Paulista. In: LEONEL et al. (Org.). Workshop sobre inovações tecnológicas em bananicultura. Botucatu: FCA/UNESP, 2008. CD-ROM.

TEIXEIRA, L.A.J.; MARTINS, A.L.M.; NATALE, W.; BETTIOL NETO, J.E. Nitrogênio e potássio em bananeira via fertirrigação e adubação convencionalatributos químicos do solo. Revista Brasileira de Fruticultura, Jaboticabal, v.29, n.1, p.143-152, 2007.

TEIXEIRA, L.A.J.; NATALE, W.; RUGGIERO, C. Nitrogen and potassium fertilization of 'Nanicão' banana (Musa AAA Cavendish subgroup) under irrigated and non-irrigated conditions. Acta Horticulturae, Leuven, v.275, p.771-779, 2002.
TEIXEIRA, L.A.J.; SPIRONELLO, A.; QUAGGIO, J.A.; FURLANI, P. Banana. In: RAIJ, B. van et al. (Ed.). Recomendações de adubação e calagem para o Estado de São Paulo. 2.ed. Campinas: IAC, 1997. p.131-132. (Boletim Técnico, 100)

THOMAS, G.W.; BERTSCH, P.M. Potassium status of temperate region soils. In: MUNSON, R.D. (Ed.). Potassium in agriculture. Madison: ASA/CSSA/ SSSA, 1985. p.131-162. 